\title{
Quando un buon insegnate fa una buona lezione? Uno studio sulle caratteristiche sociocomunicative degli insegnanti in un caso di educazione ambientale
}

Bonaiuto, Marino; Catalano, Maria; Cataldi, Silvia; D'Urso, Giulio; De Dominicis, Stefano; Faggioli, Silvia; Perucchini, Paola; Petruccelli, Irene

Published in:

Ricerche di Psicologia

DOI:

10.3280/RIP2019-001003

Publication date:

2019

Document version

Også kaldet Forlagets PDF

Document license:

Ikke-specificeret

Citation for published version (APA):

Bonaiuto, M., Catalano, M., Cataldi, S., D'Urso, G., De Dominicis, S., Faggioli, S., Perucchini, P., \& Petruccelli, I. (2019). Quando un buon insegnate fa una buona lezione? Uno studio sulle caratteristiche sociocomunicative degli insegnanti in un caso di educazione ambientale. Ricerche di Psicologia, 42(1), 43-60.

https://doi.org/10.3280/RIP2019-001003 


\title{
Quando un buon insegnate fa una buona lezione? Uno studio sulle caratteristiche sociocomunicative degli insegnanti in un caso di educazione ambientale
}

\author{
When does a good teacher make a good lesson? \\ A study on socio-communicative characteristics of teachers \\ in an environmental education program
}
Marino Bonaiuto*, Maria Catalano**, Silvia Cataldi*, Giulio D’Urso ${ }^{\circ}$, Stefano De Dominicis ${ }^{\S}$, Silvia Faggioli`, Paola Perucchini**, Irene Petruccelli ${ }^{\circ}$

\author{
*Sapienza Università di Roma, \\ Dipartimento di Psicologia dei Processi di Sviluppo e Socializzazione, \\ Via dei Marsi 78, 00185 Roma, Italia; \\ email: marino.bonaiuto@uniroma1.it; tel.: +3906 49917661 \\ email: silvia.cataldi@uniroma1.it; tel.: +3906 49917656 \\ **Università Roma Tre, Dipartimento Scienze della formazione, \\ Via del Castro Pretorio 20, 00185 Roma, Italia; \\ email: maria.catalano@uniroma3.it; tel.: +3906 57339823 \\ email: paola.perucchini@uniroma3.it; tel.: +3906 57339823 \\ 'Università degli Studi di Enna "Kore", \\ Facoltà di Scienze dell'Uomo e della Società, \\ Cittadella Universitaria, 94100 Enna, Italia; \\ email: durso.giulio@icloud.com; tel.: +39 3201609246 \\ email: i.petruccelli@icloud.com; tel.: +39 3283371576 \\ §University of Copenhagen, Department of Nutrition, Exercise and Sport, \\ Nørre Allé 51, DK-2200 Copenhagen, Danimarca; \\ email: sdd@nexs.ku.dk; tel.: +45 35326016 \\ ADI Associazione Docenti e Dirigenti Scolastici Italiani, \\ Via Vito Volterra 9, 4010 Bologna, Italia; \\ email: faggiolis@yahoo.com; tel.: +39 3357885901 \\ Indirizzo per la corrispondenza: durso.giulio@icloud.com; \\ giulio.durso@unikore.it
}

Ricevuto: 08.05.2018 - Accettato: 07.01.2019

M. Bonaiuto et al. / Ricerche di Psicologia, 2019, Vol. 42 (1), 43-60 ISSN 0391-6081, ISSNe 1972-5620 


\title{
Riassunto
}

Il presente lavoro ha lo scopo di verificare se e quali modalità didattiche e interattive messe in atto dall'insegnante in classe, durante le lezioni, possano essere un fattore legato alle buone relazioni insegnanti-discente, nonché un fattore legato allo sviluppo di proficue relazioni fra pari. I partecipanti allo studio sono stati 201 studenti. Le lezioni, svolte su temi ecologici, sono state gestite da 15 insegnanti in 3 paesi diversi. Alla lezione, inoltre, assistevano anche 17 osservatori esterni esperti, formati ad hoc per il progetto, provenienti dai diversi paesi. La valutazione delle lezioni è stata effettuata mediante un pacchetto di strumenti (Toolkit for lesson evaluation) costituito da tre diverse schede di rilevazione che hanno raccolto punti di vista diversi: osservatori esterni, studenti e docenti. Agli studenti è stato chiesto di compilare due strumenti: la SGE Student Grid e il TEP-Q Etero Evaluation. Agli osservatori esterni è stato chiesto di compilare l'SGE Observer Grid. Dalle analisi di regressione multipla emerge che la modalità di spiegazione degli insegnanti, percepita dagli osservatori, predice positivamente le adeguate relazione tra docenti e allievi. Inoltre, la comunicazione efficace dell'insegnante e l'attenzione alla relazione da parte dell' insegnate predicono le buone relazioni tra pari. Dall'analisi di correlazione, infine, emerge come le buone relazioni tra studenti sono risultate correlate significativamente con l'uso di metodologie attive da parte dell'insegnante.

Parole chiave: insegnanti, relazioni tra pari, qualità della lezione.

\begin{abstract}
The present study aims to verify if and which didactic and interactive strategies implemented by teachers in the classroom, during their lessons, can be a factor related to good teacher-student relationships, as well as a factor related to the development of satisfying relationships among peers. The participants in the study were 201 students. Lessons, conducted on ecological topic, were handled by 15 teachers in 3 different countries. In addition, 17 observers, suitably trained and coming from the different countries involved, attended the lessons. Lessons' evaluation was carried out through a toolkit (Toolkit for lesson evaluation), which consists in three different forms related to the point of view of different participants: external observers, students and teachers. Students have completed two tools: the SGE Student Grid and the TEP-Q Etero Evaluation. Observers completed the SGE Observer Grid. The multiple regression analysis suggests that teachers' explanation modality, perceived by the observers, positively predicts the good relationships between teachers and students. Furthermore, teacher's effective communication and teacher's attention to the relationship predict good relationships among peers. Finally, the correlation analysis highlights how good relations between students are related with the use of active methodologies by the teacher.
\end{abstract}

Keywords: teachers, peer relationships, quality of lesson. 


\section{Introduzione}

La relazione educativa fra insegnante e allievo è stata ampiamente discussa dalla letteratura. Molti studi si sono concentrati sul benessere scolastico inteso in termini di soddisfazione da parte degli studenti circa l'avere un insegnante con buone abilità specifiche nella trasmissione delle informazioni (e.g., Tian, Du, \& Huebner, 2015; Onwuegbuzie, Witcher, Collins, Filer, Wiedmaier, \& Moore, 2007; Zhang, 2004). Altri recenti studi suggeriscono che una relazione positiva fra insegnante e allievi risulta rilevante rispetto al grado di soddisfazione verso il docente e più in generale per la qualità della lezione, e pertanto tale relazione positiva influenza il grado di soddisfazione e la qualità della lezione stessa (e.g., Tsouloupas, Carson, \& Matthews, 2014). In altre parole, diverse evidenze empiriche mostrano come sia centrale il ruolo delle buone prassi comunicative e sociali che l'insegnate può mettere in atto per favorire nei suoi allievi adeguati livelli di benessere scolastico.

L'insegnante che vuole fungere da facilitatore nell'esperienza didattica viene definito insegnante efficace (Rogers, 1973). Le abilità che l'insegnate deve possedere riguardano soprattutto aspetti comportamentali di gestione della classe, aspetti legati alla capacità di strutturare adeguatamente la lezione grazie all'uso di metodologie attive (laboratori spaced learning e flipped classroom), nonché aspetti comportamentali legati alle modalità di comunicazione efficace verbale e non (uso dei gesti, uso dello spazio, etc.) nel migliorare anche e soprattutto il clima della classe. Tutti questi fattori possono avere un impatto sul grado di coinvolgimento della classe durante la lezione (Bonaiuto \& Maricchiolo, 2003/2009). L'instaurarsi di una buona relazione comunicativa tra le diverse figure coinvolte nel processo di apprendimento può quindi determinare un buon grado di soddisfazione sia nei docenti, ma anche e soprattutto negli studenti, poiché essa ricade sulla qualità dell'apprendimento e in particolare sulla relazione tra insegnante e allievi (Lehrer, 2015).

Un argomento molto discusso dalla letteratura, che diviene centrale nel presente contributo, sono le modalità che l'insegnante utilizza durante le lezioni e i possibili risvolti per il gruppo classe. La letteratura sottolinea, infatti, che le strategie educative messe in atto dall'insegnante durante le lezioni, nonché le sue competenze personali e sociali, talvolta favoriscono proficue relazioni insegnanti-allievi, nonché soddisfacenti relazioni fra pari, le quali nel processo di apprendimento rappresentano un fattore di protezione da rischi che minano l'adattamento scolastico e lo sviluppo psicosociale (e.g., Furrer, Skinner, \& Pitzer, 2014; Jong, Mainhard, Tartwijk, Veldman, Verloop, \& Wubbels, 2014; Kelly \& Antonio, 2016; Muscarà, Pace, Passanisi, D’Urso, \& Zappulla, 2018). 
Altri studi suggeriscono che l'uso di metodologie attive, che incentivano ad esempio gli scambi comunicativi da parte degli insegnanti, favoriscono la soddisfazione degli allievi (e.g., Zimmerman \& Paul, 2007; Galliani, 2014). Le metodologie didattiche attive quindi mettono al centro del processo educativo e di apprendimento gli allievi - coinvolgendo la loro creatività e -favorendo il loro senso di iniziativa - senza prescindere naturalmente dai contenuti curricolari che sono sempre centrali nel processo. Ciò implica, pertanto, la funzione attiva degli allievi i quali, anche in una semplice situazione di confronto post-lezione, possono così mettersi maggiormente in gioco in un processo di trasmissione di contenuti che sia maggiormente dialettico; il fare esperienza attiva in classe, sollecitati dalle abilità dell'insegnate, può incentivare anche il dialogo fra gli allievi appartenenti alla classe stessa (Muijs \& Reynolds, 2017).

Molti studi, infatti, sottolineano come tecniche che incentivavo il dialogo tra gli studenti e il dialogo insegnante-allievo possono incrementare la soddisfazione del clima scolastico (e.g., Dukuzumuremyi \& Siklander, 2018; Šejtanić1i \& Ilić, 2016). In questa prospettiva, l'azione educativa dell'insegnante, nonché la scelta delle metodologie didattiche da usare, si configura come variabile significativa anche per lo sviluppo sociale degli allievi. In altre parole, lo scenario educativo diventa luogo chiave nello sviluppo di competenze socio-relazionali. In particolare, l'insegnante assolve a una doppia valenza: da un lato, favorisce l'acquisizione di competenze, mettendo in atto strategie educative efficaci per la relazione insegnante-allievo; dall'altro lato, favorisce l'implementazione di buone prassi nelle relazioni fra pari. Queste ultime, soprattutto nello sviluppo, oltre a potere giocare un ruolo nell'apprendimento, si configurano come un fattore di protezione per il benessere individuale e sociale (Pace, D'Urso, \& Zappulla, 2018).

Orpinas e Horne (2006), in tal senso, nel modello concettuale denominato "School Social Competence Development", individuano proprio la figura dell'insegnante, con le sue competenze, come una componente fondamentale nella creazione di un buon clima di classe. Come suggeriscono La Paro e Pianta (2003), un clima di classe ottimale è caratterizzato da uno stile comunicativo, tra insegnante-allievo e tra allievi, fatto di supporto, nonché intriso da scambi che incentivano il dialogo, sollecitando la reattività di tutti gli attori coinvolti nel processo educativo. L'insegnante, quindi, quando sceglie le proprie metodologie, dovrebbe pensare ai bisogni individuali e sociali dell'intera classe.

Altri studi, che si sono concentrati più sul ruolo dell'insegnante, suggeriscono come le metodologie didattiche scelte e usate dall'insegnante possono anche rivestire un ruolo importante al fine di incrementare l'efficacia dell'insegnamento con gli studenti, nonché incentivando proficue relazio- 
ni tra insegnanti-allievi (Hodge, 2014; Zeng, Benner, \& Silva, 2016). Grazie alle prassi educative che smussano le relazioni asimmetriche insegnante-allievo, l'esperienza in classe per gli allievi diviene positiva poiché gli insegnanti incrementano la loro autoefficacia, nonché divengono per gli studenti fonte sicura su cui attingere per acquisire conoscenze e buone prassi di regolazione socio-emotiva. In tale senso, la teoria di Bandura e gli studi a essa ispirati (Bandura, 1997; Bruce \& Ross, 2008; Bruce, Esmonde, Ross, Dookie, \& Beatty, 2010; Goddard, Hoy, \& Woolfolk Hoy, 2004; Tschannen-Moran \& Woolfolk Hoy, 2001; Tschannen-Moran, Wolfolk Hoy, \& Hoy, 1998) mostrano come gli insegnanti con un'elevata efficacia sentono di poter influire positivamente sulla scelta delle metodologie da usare per incentivare la creazione di condizioni adeguate e ottimali per la soddisfazione degli alunni (Woolfolk Hoy \& Weinstein 2006). Diverse ricerche hanno, infatti, messo in luce che gli insegnanti con alti livelli di autoefficacia e credenze educative di tipo costruttivista tendono a utilizzare una maggior varietà di metodologie didattiche e forniscono supporto e al tempo stesso autonomia ai loro studenti (Fives, \& Alexander, 2004; Heneman, Kimball, \& Milanowski, 2006). In tal senso, infatti, lo studio di Bruce e colleghi (2010), che ha valutato un programma di apprendimento a cui hanno partecipato studenti e insegnanti, sottolinea che l'efficacia degli educatori risulta di fondamentale importanza per l'apprendimento e più in generale dunque per la relazione educativa insegnante-allievo. In questo modo la percezione dell'allievo sul proprio insegnante sarà maggiormente positiva, perché lo sentirà più vicino ai suoi bisogni psicoeducativi e questi ultimi si riverseranno anche sulle relazioni che l'adolescente instaura con i propri pari.

Inoltre, anche gli studiosi della comunicazione suggeriscono che gli insegnanti che fanno un buon uso della prossemica, che fanno un buon uso dello spazio delle metodologie attive, hanno maggiori probabilità di riportare successi, in termini di soddisfazione da parte della propria classe circa la relazione insegnate-allievo, nonché in termini di relazioni tra pari (e.g., Bonaiuto \& Maricchiolo, 2003/2009; Brophy \& Good, 1970; Monroe, Andrews, \& Biedenweg, 2008; Watzlawick, Beavin, \& Jackson, 1971). In tal senso, le azioni didattiche hanno peso sia sulle relazioni verticali (solitamente rigide tra insegnate-allievo) sia su quelle orizzontali (relazioni tra pari).

\section{Obiettivio e ipotesi}

Considerando la natura transazionale della comunicazione umana, nonché l'importanza della soddisfazione scolastica legata alla relazione insegnante-allievi e quella tra compagni di classe, il presente contributo ha lo 
scopo di verificare se le modalità di gestione della classe adottate dagli insegnanti durante le lezioni possono essere considerate un fattore che influisce sulla soddisfazione degli studenti circa vari aspetti della lezione stessa, dell'insegnante, e delle relazioni fra pari e fra insegnanti e allievi.

Tale studio si inserisce nell'ambito di un progetto di innovazione didattica che ha visto coinvolti insegnanti e studenti di scuole secondarie in Polonia, Turchia e Italia e alcuni ricercatori.

In particolare, il presente studio indagherà, nell'ottica della teoria ecologica di Bronfenbrenner (1979), i seguenti aspetti: la gestione della classe (strategie educative, modalità di presentazione dei contenuti, abilità prosociali, comunicazione, attenzione per le relazioni, ecc.) valutata dagli osservatori e dagli studenti stessi, nonché la relazione studenti e insegnanti e tra studenti valutata dagli studenti.

In linea con la letteratura (e.g., Tsouloupas, Carson, \& Matthews, 2014), ci aspettiamo che le modalità di gestione della classe, nei suoi vari aspetti, messe in atto dagli insegnanti predicano relazioni adeguate e soddisfacenti tra insegnanti e allievi (ipotesi 1). Questa ipotesi verrà testata considerando il punto di vista dell'osservatore esterno che può svolgere un ruolo di valutatore neutrale e quindi avere una percezione consensuale della gestione della classe. Al contrario la relazione insegnate-allievo verrà valutata dagli studenti.

Inoltre, alla luce dei più rilevanti contributi teorici della letteratura (Bruce et al., 2010), ipotizziamo che la percezione degli studenti in merito alle pratiche educative messe in atto dagli insegnanti (comunicazione efficace, autoregolazione, attenzione alla relazione, cura delle attività e metodologie attive così come percepite dagli studenti) influenzino le buone relazioni tra pari così come percepite dagli studenti stessi (ipotesi 2).

Infine, ci aspettiamo, in linea con la letteratura (e.g., Muijs \& Reynolds, 2017), che la gestione della classe (metodologie attive da parte dell'insegnante, l'uso dello spazio e la presentazione dei contenuti da parte dell'insegnante) valutata dagli osservatori esterni predica soddisfacenti relazioni tra pari valutate dagli studenti (ipotesi 3).

\section{Partecipanti e procedura}

I partecipanti allo studio sono 201 studenti provenienti dalle scuole medie superiori coinvolte nel progetto, di età compresa fra i 15 e i 19 anni $(\mathrm{M}=16,36 ; \mathrm{DS}=1,10)$, che hanno partecipato alle lezioni. Il $36,8 \%$ dei partecipanti sono maschi e il $63,2 \%$ femmine. Inoltre, sono stati coinvolti 17 osservatori esperti (provenienti da Sapienza Università di Roma) e altri non esperti, formati ad hoc. Ad ogni lezione assistevano un numero di osservatori compreso tra 11 e 17 . Gli osservatori si trovavano nella stessa 
stanza dove si svolgeva la lezione, in uno spazio limitato a parte, ma avevano libertà di movimento.

Il progetto ha previsto lo svolgimento di lezioni su temi ecologici (e.g., workshop sul ciclo del carbone, riciclo dei materiali, acquisto responsabile) in 3 Paesi diversi. Nello specifico, tre lezioni sono state svolte in Polonia, due in Turchia e quattro in Italia. Le lezioni sono sempre state condotte da insegnanti in coppia provenienti dallo stesso paese davanti a una platea di studenti provenienti dai diversi paesi coinvolti dal progetto (ciascuna lezione ha avuto un numero di studenti partecipanti compreso tra un minimo di 12 e un massimo di 30 persone). Gli insegnanti coinvolti erano 18 e sono stati reclutati sulla base di un'intervista aperta circa le motivazioni a partecipare, la conoscenza della lingua inglese, nonché l'interesse nei confronti di tematiche ambientali e le competenze didattiche all'uso di metodologie attive. In particolare, potevano essere utilizzate oltre alle metodologie formative classiche di tipo frontale anche lezioni di tipi dialogico, laboratoriale e in gruppo, spaced learning e flipped classroom.

Tutti i partecipanti (compresi gli insegnanti) hanno trascorso un'intera settimana insieme venendo coinvolti oltre alle lezioni, anche in gite e altre attività ricreative. Successivamente al primo incontro tutti i partecipanti sono stati invitati a entrare in relazione tra di loro anche in chat e reti sociali (Facebook, Istagram e WhatsApp).

L'addestramento all'uso degli strumenti è avvenuto durante una giornata di formazione all'inizio del progetto, nonché in occasione di ogni rilevazione, per un totale di 4 incontri di formazione.

Gli studenti e gli insegnanti partecipanti alla ricerca sono stati reclutati su base volontaria, previa spiegazione della ricerca stessa, nonché previo consenso informato e autorizzazione dei dirigenti scolastici. Le procedure di somministrazione dei questionari sono state svolte rispettando gli standard etici della ricerca scientifica nel contesto nazionale pertinente, garantendo il pieno rispetto della privacy secondo la normativa nazionale di riferimento.

Durante lo svolgimento delle lezioni da parte degli insegnanti, gli osservatori valutavano le attività didattiche, le modalità di comunicazione $\mathrm{e}$ le caratteristiche degli insegnanti, utilizzando una griglia di osservazione appositamente creata. Alla fine della lezione, a ciascun/a studente/essa veniva chiesto di esprimersi circa la lezione, l'insegnante e le relazioni, utilizzando un questionario appositamente creato e un questionario standardizzato (PET-Q etero-valutativo, vedi paragrafo Strumenti). Anche gli insegnanti venivano invitati a compilare un analogo questionario (TEP-Q auto-valutativo) inerente se stessi e la loro lezione, al termine della stessa ${ }^{1}$.

I risultati relativi alle autovalutazioni degli insegnanti non sono stati considerati in que- 
Gli strumenti venivano somministrati nella versione in lingua inglese per poter essere compilati da persone provenienti dai tre Paesi partecipanti al progetto (Italia, Polonia e Turchia).

\section{Strumenti}

La valutazione delle lezioni si è servita di un pacchetto di strumenti (Toolkit for lesson evaluation) costituito da tre diverse schede di rilevazione che hanno raccolto i punti di vista diversi di osservatori esterni e studenti. Agli studenti è stato chiesto di compilare due strumenti: la SGE Student Grid e il TEP-Q Etero Evaluation; mentre gli osservatori hanno compilato la $S G E$ Observer Grid.

La SGE Student Grid (creata ad hoc dagli autori del contributo per la ricerca in questione) è uno strumento costruito ad hoc composto da 44 item articolati in sezioni distinte:

1) la sezione Lesson ( $\alpha=0,847)$, che intende indagare come è stata percepita la lezione (item creati ad hoc);

2) la sezione Teacher $(\alpha=0,773)$, che riguarda le capacità dell'insegnante (item creati ad hoc);

3) la sezione relativa alla percezione delle caratteristiche personali e interpersonali dell'insegnante comprende 3 scale: Personality $(\alpha=.757)$ comprende 5 item che ricalcano le dimensioni di personalità dei Big Five - Vecchione, Alessandri, Barbaranelli, \& Caprara, 2010; Reputation $(\alpha=.833)$ comprende 4 di reputazione che ricalcano gli item del Reputation Institute, Van Riel, \& Fombrum, 2007; Interpersonal perception of the teacher $(\alpha=.712)$ comprende 2 item che ricalcano le dimensioni di percezione interpersonale Fiske, Cuddy, \& Glick, 2008);

4) la sezione Relations ( $\alpha=0,738$ ), che si focalizza sulle relazioni instaurate nel corso della lezione (13 item costruiti ad hoc). Nello specifico, questa sezione è composta da due scale: Relations 1 che riguarda il rapporto con i pari (relazioni orizzontali) $(\alpha=0,508)$. Gli item che maggiormente contribuiscono alla costruzione del fattore sono il numero 8 (I felt good about being a member of this class) e il numero 9 (I felt comfortable being with the other students in this class); mentre contribuisce negativamente l'item 13 (I felt I was not a very important person in this class). La scala Relations 2 riguarda, invece, il rapporto con l'insegnante (relazioni verticali) $(\alpha=0,678)$. Gli item che maggiormente contribuiscono alla costruzione del fattore sono il numero 3 (My teacher was upset when I helped who was stuck with a problem) e il numero 10 (The teacher got upset when students in this class tried to help each other);

5) la sezione Climate in the classroom ( $\alpha=0,735)$, che indaga il clima di classe (4 item costruiti ad hoc). Per ciascun item, gli studenti devono

sta sede. 
esprimere la propria valutazione su una scala Likert a 5 passi $(1=$ per niente/mai; 5 = completamente/sempre).

I 1 TEP-Q Etero-Evalution (Teacher's Educational Practicies Questionnaire, Catalano, Perucchini, \& Vecchio, 2014) è uno strumento che permette di valutare le pratiche educative di gestione della classe messe in atto dall'insegnante durante le lezioni svolte. Il questionario è composto da 36 item per i quali bisogna esprimere la frequenza di messa in atto del comportamento su una scala Likert a 6 passi $(0=$ mai; $5=$ sempre $)$. Precedenti studi di validazione (Catalano et al., 2014) hanno evidenziato la presenza di cinque sottoscale: la Comunicazione Efficace fa riferimento alle pratiche educative relative alla comunicazione verbale, paraverbale e non verbale; l'Autoregolazione Comunicativa, indica la capacità di controllare i propri stati emotivi attraverso la comunicazione; la sottoscala Metodologie attive è relativa all'uso di metodologie alternative alla lezione frontale tradizionale (lavori di gruppo, tutoring tra pari, ecc..); Cura nelle attività riguarda la capacità di organizzare le attività in classe; infine l'Attenzione alle relazioni fa riferimento alla capacità di considerare le dinamiche di gruppo nella classe.

Agli osservatori esterni è stato chiesto di compilare l'SGE Observer Grid, uno strumento creato ad hoc per permettere di valutare l'insegnante durante lo svolgimento della sua lezione. La scheda è composta da tre sezioni.

1) La sezione Classroom Management considera 8 aspetti legati alla gestione della classe (item costruiti ad hoc sulla base di D'Alonzo, 2012): educational strategies and teaching methods (8 item); use of the space (4 item); activities management (7 item); content and instruction presentation (10 item); discussion management (9 item); feedback (10 item); prosocial abilities (7 item); strenghts and weaknesess (6 item).

2) La sezione Bodily Communication, è dedicata ad analizzare la comunicazione corporea dell'insegnante, considerando 8 aspetti (item costruiti ad hoc sulla base di Bonaiuto \& Maricchiolo, 2003/2009): proxemics (8 item); postural and physical orientation (9 item); voice rhythm and tone (8 item); gaze (7 item); facial expressions (18 item); hand gestures (6 item); physical cues (5 item); head, arms, chest, legs movements (4 item).

3) La sezione Interpersonal Characteristics è focalizzata sulle caratteristiche personali e sociali dell'insegnante ed è composta da 4 aspetti: personality $(5$ item che ricalcano le dimensioni di personalità dei Big Five, Vecchione, Alessandri, Barbaranelli, \& Caprara, 2010); reputation (4 item di reputazione che ricalcano gli item del Reputation Institute, Van Riel \& Fombrum, 2007); interpersonal perception (2 item 
che ricalcano le dimensioni di percezione interpersonale Fiske, Cuddy, \& Glick, 2008); climate in the classroom (4 item costruiti ad hoc).

Per ciascun aspetto delle tre sezioni gli osservatori dovevano attribuire un punteggio su una scala Likert a 5 passi $(1=$ mai; $5=$ sempre $)$ ed espri mere una valutazione generale scegliendo uno "smile" tra tre proposte, recanti rispettivamente un'espressione triste, neutra o felice. Era inoltre possibile scrivere delle annotazioni libere durante l'osservazione.

Di seguito vengono riportati i valori dei coefficienti alfa di Cronbach delle dimensioni delle sezioni compilate dagli osservatori: nella sezione Classroom Management, Educational Strategies 0,829, Use of the space 0,576, Activity management 0,68, Presentation of contents and istructions 0,831, Discussion management 0,941 , Feedback 0,635 , Prosocial activities 0,905, Strenghts 0,775; nella sezione Bodily Communication: Proxemic 0,520, Voice rhythm, volume and tone 0,629, Gaze 0,887, Facial expression 0,788, Hand gestures 0,755 ; nella sezione Interpersonal Charcateristics: Communion 0,647, Agency 0,626, Reputation 0,864, Climate 0,864 .

\section{Analisi dei dati}

Le analisi dei dati sono state svolte con SPSS versione 22. In particolare, sono state effettuate delle analisi di regressione e correlazioni volte a testare le tre ipotesi.

\section{Risultati}

Nella prima analisi di regressione multipla, effettuata per testare l'ipotesi 1 , abbiamo inserito come variabile criterio la relazione con gli insegnanti valutata dagli studenti (Relation to Teachers) e come variabili indipendenti le variabili di Classroom Management: strategie educative, modalità di presentazione dei contenuti, abilità prosociali e punti di forza $\mathrm{e}$ debolezza (senso dell'umorismo, creatività e entusiasmo), valutate dagli osservatori esterni, mediante la scala SGE Observer Grid. Dall'analisi emerge un modello significativo $\left(R^{2}=0,34, F(1,9)=2,036, p<0,05\right)$. Nello specifico, la variabile che è risultata significativa sulla relazione degli studenti con gli insegnanti è la modalità di presentazione di contenuti e istruzioni da parte dell' insegnante $(\beta=-1,01 ; p=0,05)$ : più gli osservatori criticano la capacità degli insegnanti di porre attenzione all'assegnazione di compiti e regole comuni, meno positivamente sarà valutata la relazione insegnate e studente. In altre parole, più l'insegnante, secondo gli osservatori, è stata chiara ed efficace nell'esporre i contenuti e le istruzioni per le attività da svolgere, migliore sarà la relazione tra insegnante e allievo, valutato da quest'ultimo. Ciò significa che una buona relazione con gli studenti si stabilisce quando l'insegnante è chiara nel presentare i contenuti 
della lezione e le istruzioni, parla in modo scorrevole, dando enfasi alle parole importanti e usando come supporto oggetti e lavagna, piuttosto che quando usa un linguaggio esitante e con degli errori.

Nella seconda analisi di regressione, effettuata per testare l'ipotesi 2, abbiamo incluso la variabile "relazioni tra pari" come criterio e come predittori le cinque dimensioni del TEP-Q (comunicazione efficace, autoregolazione, attenzione alla relazione, cura delle attività e metodologie attive così come percepite dagli studenti). Dall'analisi svolta emerge un modello significativo $\left(R^{2}=0,71, F(1,9)=4,869, p<0,05\right)$. Nello specifico, le variabili che risultano predittori significativi sulle relazioni positive fra pari sono la comunicazione efficace dell'insegnante $(\beta=3,2 ; p=0.04) \mathrm{e}$ l'attenzione alle relazioni $(\beta=2,4 ; p=0,05)$. Dunque, maggiore è la percezione degli studenti circa l'efficacia della comunicazione e la cura della relazione da parte dell'insegnante, migliore saranno le relazioni tra pari, valutate dagli studenti.

In riferimento all'ipotesi 3 , inserendo come predittori le variabili "uso di metodologie attive da parte dell'insegnante", "uso dello spazio" e "presentazione dei contenuti da parte dell'insegnante" e come criterio la variabile "relazioni tra pari", emerge un modello, tendenzialmente significativo $\left(R^{2}=0,53, F(1,9)=3,99, p=0,08\right)$, con la variabile "uso di metodologie attive da parte dell'insegnante" come unico predittore, appunto, tendenzialmente significativo $(\beta=0,56 ; p=0,09)$. Per approfondire tale tenden$\mathrm{za}$, è stata effettuata una analisi della correlazione tra la relazionale fra gli studenti (Relation to Schoolmates), valutata dagli studenti, con le scale relative alla Classroom Management, compilate dagli osservatori esterni. Dall' analisi emerge come le buone relazioni tra studenti sono risultate correlate significativamente con l'uso di metodologie attive da parte dell'insegnante $(r=0,70 ; \mathrm{p}<0,001)$. Pertanto l'uso delle metodologie attive favoriscono lo stabilirsi di relazioni positive tra studenti.

\section{Discussioni e conclusioni}

Lo studio ha voluto individuare le variabili che, nel contesto educativo, abbiano un impatto sulla qualità della gestione della classe, sulla relazione con l'insegnante e sulle relazioni fra pari (variabili valutate dallo studente dopo aver assistito a lezioni su tematiche ecologiche da parte dei docenti).

Il presente contributo mira a individuare gli indicatori di "buone prassi" didattiche, con attenzione particolare alle modalità più congrue da utilizzare all'interno della classe per facilitare la soddisfazione al fine di creare e valutare modelli educativi innovativi evidence based.

In particolare, a conferma dell'ipotesi, dalle analisi emerge che la modalità di esposizione dei contenuti degli insegnanti, percepita dagli osservatori, ha un impatto positivo sulla relazione tra docenti e allievi, al contrario di fattori quali le strategie educative e le abilità prosociali che non sono risultati predittori significativi come invece dovrebbero secondo nu- 
merosi studi (e.g., Bandura, 1997; Dozza, 2006; McGinnis \& Goldstein, 1997; Mitchell, 2014). In particolare, quindi, la relazione allievo-docente risulta più positiva quando le spiegazioni dei docenti sono anzitutto chiare, ovvero quando sono precedute da istruzioni anch'esse chiare ed i contenuti sono illustrati con il supporto di oggetti e lavagna. Questo risultato evidenzia l'importanza che assumo l'esplicitazione dei diversi aspetti relativi alla lezione e la chiarezza, all'interno della relazione docente - discente. Questo dato può essere anche considerato particolarmente rilevante, poiché si basa sul giudizio di un osservatore esperto super partes e quindi non direttamente coinvolto nella relazione educativa.

A conferma dell'ipotesi 2, emerge che la percezione positiva degli studenti circa le pratiche educative messe in atto dagli insegnati risulta positivamente connessa con l'incremento delle relazioni tra pari. Dunque, in linea con quanto suggerito dalla letteratura (e.g., Dozza, 2006; Tsouloupas, Carson, \& Matthews, 2014; Oxford, 2016), è possibile affermare che un insegnante percepito come attento alle relazioni con gli studenti ed efficace nella comunicazione con il gruppo classe possa avere sullo stesso effetti benefici dal punto di vista socio-relazionale. In altre parole, un docente nella prassi educativa non solo trasmette informazioni relative agli argomenti, ma comunica implicitamente agli studenti delle competenze sociali e comunicative, utili per i contesti di gruppo. In un'ottica didattica l'insegnate dovrebbe quindi essere pronto e sufficientemente adeguato nella gestione delle relazioni sociali, nonché avere cura dell'aspetto comunicativo nella gestione del gruppo. Infatti, l'educatore nel processo di insegnamento non svolge un ruolo limitato all'acquisizione dei saperi, ma costituisce il fattore chiave per generare reti sociali nei suoi allievi. Questo risultato conferma l'importanza del ruolo dell'insegnante come facilitatore di relazioni all'interno della classe. In tal senso, la relazione con il docente non assume la configurazione classica di relazione gerarchica, ma di relazione che contribuisce, attraverso la sua azione, a favorire non solo l'apprendimento, ma anche proficue relazioni tra i pari. L'attenzione del docente quindi non deve spostarsi dall'insegnamento dei saperi alla relazione, ma deve riuscire a muoversi lungo un doppio binario che una volta percorso porti a due esiti: da un lato, l'apprendimento dei contenuti e delle competenze specifiche attivate dalla lezione, dall'altro, lo sviluppo di competenze prosociali in classe (e.g., Tsouloupas, Carson, \& Matthews, 2014).

In merito alla verifica dell'ipotesi 3 , le analisi suggeriscono che le metodologie attive messe in atto degli insegnanti e così percepite dagli studenti risultano fattori connessi nonché facilitanti le positive relazioni in classe. Quest'ultimo si traduce non soltanto nell'acquisizione di nozioni teoriche, ma indirettamente anche nella trasmissione di strategie che influiscono sulla capacità di saper fare e nel saper essere in relazione degli 
studenti. Tale risultato è in linea con la letteratura, che evidenzia come l'uso adeguato delle metodologie didattiche attive favorisca un clima positivo e proficuo all'apprendimento (Evertson, 1994). Dunque, questo risultato suggerisce come le buone prassi scolastiche dovrebbero mirare all'uso di metodologie innovative per una completa formazione dei docenti su quelli che risultano essere i canali della comunicazione e sull'importanza delle relazioni umane nei contesti di gruppo (Alberts, Nakayama, \& Martin, 2015). L'intervento dell'insegnante, come facilitatore delle relazioni orizzontali, è fondamentale per creare una lezione didatticamente efficace mirata all'apprendimento di competenze e contenuti scolastici e allo sviluppo di competenze socio-relazionali, implicitamente derivate dall'uso di strategie didattiche attive. In tal senso, mettere in discussione le proprie metodologie per l'insegnamento risulta funzionale per creare una scuola inclusiva, che favorisca il dialogo e il confronto fra pari (Petruccelli \& Fabrizi, 2005).

Il presente contributo ci permette di rilevare come un buon insegnante debba possedere non solo qualità didattiche, ma anche competenze sociali e di comunicazione efficace. In altre parole, la trasmissione delle informazioni può, in qualche modo, essere influenzata dalle modalità con cui viene trasmesso il messaggio in termini di contenuti. Ed è proprio questa condizione ottimale che favorisce l'apprendimento scolastico e per riflesso il benessere e il funzionamento del gruppo classe (Castoldi, 2010).

Dunque, emerge la necessità di creare modelli educativi che utilizzino prassi e metodologie attive che non facciano solo attenzione alla trasmissione dei contenuti, ma che abbiano come obiettivo quello di favorire atteggiamenti positivi fra gli studenti attraverso strategie educative specifiche (Caprara, Gerbino, Luengo Kanacri, \& Vecchio, 2014; Margiotta, 1999).

Come osservano Orpinas e Horne (2006) l'efficacia nell'insegnamento include non solo l'ampiezza e la profondità della conoscenza della materia, ma anche la padronanza delle abilità nel trasmettere questa conoscenza agli allievi, motivandoli ad apprendere. Va sottolineato, come talvolta gli insegnanti facciano fatica ad utilizzare metodologie attive per trasmettere contenuti scolastici e abilità sociali. Flay (2002), inoltre, suggerisce come sia importante adottare, da parte degli insegnanti, un approccio globale che promuova non solo il comportamento positivo, ma che curi anche il rendimento scolastico degli allievi con prassi opportune.

Alcuni limiti vanno considerati nell'interpretazione dei risultati del presente studio. Un primo limite è la metodologia di reclutamento dei partecipanti: è stato utilizzato un campione di convenienza che rende difficile la generalizzazione dei risultati. Un secondo limite è quello legato alla desiderabilità sociale, legata all'uso di questionari autosomministrati. È pos- 
sibile cioè che gli studenti abbiano sopravvalutato le competenze degli insegnanti. Studi futuri potrebbero anche prevedere l'uso di metodologie implicite. Un ulteriore limite riguarda il setting e il disegno sperimentale: ulteriori approfondimenti potrebbero mirare a migliorare la qualità del setting, incrementando la formazione degli insegnanti e degli osservatori e creando ulteriori spazi e tempi di conoscenza tra gli allievi, nonché aggiungendo un gruppo di controllo per verificare l'efficacia dell'intervento. Infine, si sottolinea al fatto che le lezioni si svolgevano in lingua inglese, che non è la lingua di origine degli studenti e degli insegnanti.

Concludendo, il presente studio ha confermato empiricamente, in diverse classi di media superiore di tre paesi europei, il valore predittivo di alcune variabili (modalità di presentazione dei contenuti da parte dell'insegnante, la comunicazione efficace, l'attenzione alle relazioni, l'uso di metodologie attive da parte dell'insegnante) riconosciute in letteratura come significative nella creazione di adeguate relazioni fra pari, nonché connesse alla soddisfazione scolastica in termini di competenze sociali. L'importanza di questi risultati riguarda anche l'aver preso in considerazione la prospettiva della figura dell'osservatore esterno, il quale consente di accedere ad una valutazione terza nel setting scolastico; inoltre, le lezioni sono state espletate grazie all'uso di metodologie attive diverse e innovative. In futuro, si potrebbero approfondire ulteriori aspetti nella relazione educativa, studiando ad esempio anche le caratteristiche individuali relative a insegnanti e studenti, per verificare possibili effetti di mediazione e/o moderazione. Nello specifico, si ritiene che la ricerca debba anche informare gli attori del processo educativo, per renderli consapevoli sull'importanza del loro ruolo e dei suoi effetti sui gruppi scolastici, per incrementare i loro punti di forza e lavorare su eventuali punti di debolezza, nonché per costruire programmi che promuovano l'inclusione e la cooperazione come fattori di protezione nelle relazioni fra pari.

\section{Note}

La ricerca è stata effettuata all'interno del progetto "SGE: Science and Global Education beyond the barriers of learning difficulties" finanziato nel bando e schema di finanziamento Programma Erasmus+ Call 2015 - KA2 - Cooperation for Innovation and the Ex change of Good Practices - Strategic Partnerships for school education (progetto n. 20151-IT02-KA201-014774, durata 24 mesi, 01/10/2015 - 30/09/2017).

Il contributo è inoltre il risultato di un lavoro di co-creazione nel quadro del progetto "ACCOMPLISSH: Accelerate co-creation by setting up a multi-actor platform for impact from Social Sciences and Humanities" nel bando EC Horizon 2020 Call: H2020-REFLECTIVE-SOCIETY-2015 Topic: REFLECTIVE-11-2015 Type of action: CSA (Grant Agreement n. 693477, durata 36 mesi, 01/03/2016 - 28/02/2019).

L' articolo è frutto del lavoro congiunto degli autori. Per mere ragioni di attribuzione, si specifica che Silvia Cataldi è autrice delle sezioni: Procedura e partecipanti; Analisi dei dati. 


\section{Riferimenti bibliografici}

Alberts, J. K., Nakayama, T. K., \& Martin, J. N. (2015). Human communication in society. UK: Pearson.

Bandura, A. (1997). Self-efficacy: The exercise of control. New York: W.H. Freeman.

Bonaiuto, M., \& Maricchiolo, F. (2003/2009). La comunicazione non verbale (seconda edizione). Roma: Carocci.

Bronfenbrenner, U. (1979). The ecology of human development. Cambridge MA: Harvard University Press.

Brophy, J. E., \& Good, T. L. (1970). Teachers' communication of differential expectations for children's classroom performance: Some behavioral data. Journal of educational psychology, 61(5), 365-374. DOI: 10.1037/h0029908.

Bruce, C. D., \& Ross, J. A. (2008). A model for increasing reform implementation and teacher efficacy: teacher peer coaching in grade 3 and 6 mathematics. Canadian Journal of Education, 31(2), 346-370. DOI: 10.2307/20466705.V

Bruce, C. D., Esmonde, I., Ross, J., Dookie, L., \& Beatty, R. (2010). The effects of sustained classroom-embedded teacher professional learning on teacher efficacy and related student achievement. Teaching and Teacher Education, 26(8), 1598-1608. DOI: 10.1016/j.tate.2010.06.011.

Caprara, G. V., Gerbino, M., Luengo Kanacri, P., \& Vecchio, G. (2014). Educare alla prosocialità. Teoria e buone prassi. Milano: Pearson Italia.

Castoldi, M. (2010). Didattica generale. Milano: Mondadori Università.

Catalano, M. G., Perucchini, P., \& Vecchio, G. M. (2014). The quality of teachers' educational practices: internal validity and applications of a new self-evaluation questionnaire. Procedia-Social and Behavioral Sciences, 141, 459464. DOI: 10.1016/j.sbspro.2014.05.080.

D’Alonzo, L. (2012). Come fare per gestire la classe nella pratica didattica. Firenze: Giunti.

Dozza, L. (2006). Relazioni cooperative a scuola: il lievito e gli ingredienti. Trento: Edizioni Erickson.

Dukuzumuremyi, S., \& Siklander, P. (2018). Interactions between pupils and their teacher in collaborative and technology-enhanced learning settings in the inclusive classroom. Teaching and Teacher Education, 76, 165-174. DOI: 10.1016/j.tate.2018.08.010.

Evertson, C. M. (1994). Classroom management for elementary teachers. Boston: Allyn \& Bacon.

Fiske, S. T., Cuddy, A. J. C., \& Glick, P. (2007). Universal dimensions of social cognition: warmth and competence. Trends in Cognitive Science, 11, 77-83. DOI: $10.1016 /$ j.tics.2006.11.005.

Fives, H., \& Alexander, P. A. (2004). Modeling teachers' efficacy, knowledge, and pedagogical beliefs. Paper presented at the annual meeting of the American Psychological Association. Honolulu, HI.

Flay B.F. (2002). Positive youth development requires comprehensive health promotion programs. American Journal of Health Behaviour, 26, 407-424. DOI: 10.5993/AJHB.26.6.2. 
Furrer, C. J., Skinner, E. A., \& Pitzer, J. R. (2014). The influence of teacher and peer relationships on students' classroom engagement and everyday motivational resilience. National Society for the Study of Education, 113(1), 101123.

Galliani, L. (2014). Formazione degli insegnanti e competenze nelle tecnologie della comunicazione educativa. Italian Journal of Educational Research (23), 93-103.

Goddard, R. D., Hoy, W. K., \& Woolfolk Hoy, A. (2004). Collective efficacy beliefs: theoretical developments, empirical evidence, and future directions. $E d$ ucational Researcher, 33(3), 3-13. DOI: 10.3102/0013189X033003003.V

Heneman, H. G., III, Kimball, S., \& Milanowski, A. (2006). The Teacher Sense of Efficacy Scale: Validation Evidence and Behavioral Prediction. WCER Working Paper No. 2006-2007. Madison: University of Wisconsin-Madison, Wisconsin Center for Education Research .

Hodge, B. (2014). Teaching as communication. London, UK: Routledge.

Jong, R., Mainhard, T., Tartwijk, J., Veldman, I., Verloop, N., \& Wubbels, T. (2014). How pre-service teachers' personality traits, self-efficacy, and discipline strategies contribute to the teacher-student relationship. British Journal of Educational Psychology, 84(2), 294-310. DOI: 10.1111/bjep.12025.

Kelly, N., \& Antonio, A. (2016). Teacher peer support in social network sites. Teaching and Teacher Education, 56, 138-149. DOI: 10.1016/j.tate.2016.02.007.

La Paro, K.M., \& Pianta, R.C. (2003). CLASS: classroom assessment scoring system. Charlottesville: University of Virginia Press.

Lehrer, K. (2015). Theory of knowledge. UK: Routledge.

Margiotta, U. (Ed.). (1999). L'insegnante di qualità. Valutazione e performance. Roma: Armando Editore.

McGinnis, E., \& Goldstein, A. P. (1997). Skillstreaming the elementary school child: New strategies and perspectives for teaching prosocial skills. USA: Research Press.

Mitchell, D. (2014). What really works in special and inclusive education: Using evidence-based teaching strategies. UK: Routledge.

Monroe, M. C., Andrews, E., \& Biedenweg, K. (2008). A framework for environmental education strategies. Applied Environmental Education \& Communication, 6(3-4), 205-216. DOI: 10.1080/15330150801944416.

Muijs, D., \& Reynolds, D. (2017). Effective teaching: Evidence and practice. New York: Sage.

Muscarà, M., Pace, U., Passanisi, A., D’Urso, G., \& Zappulla, C. (2018). The Transition from Middle School to High School: The Mediating Role of Perceived Peer Support in the Relationship between Family Functioning and School Satisfaction. Journal of Child and Family Studies, 27(8), 2690-2698. DOI: 10.1007/s10826-018-1098-0.

Onwuegbuzie, A. J., Witcher, A. E., Collins, K. M., Filer, J. D., Wiedmaier, C. D., \& Moore, C. W. (2007). Students' perceptions of characteristics of effective college teachers: a validity study of a teaching evaluation form using a mixed-methods analysis. American Educational Research Journal, 44(1), 113160. DOI: $10.3102 / 0002831206298169$. 
Orpinas, P., \& Horne, A. M. (2006). Bullying prevention: Creating a positive school climate and developing social competence. Washington, D.C.: American Psychological Association.

Oxford, R. L. (2016). Teaching and researching language learning strategies: Self-regulation in context. UK: Taylor \& Francis.

Pace, U., D’Urso, G., \& Zappulla, C. (2018). Negative eating attitudes and behaviors among adolescents: The role of parental control and perceived peer support. Appetite, 121, 77-82. DOI: 10.1016/j.appet.2017.11.001.

Petruccelli, I., \& Fabrizi, A. (2005). Orientarsi per non disperdersi. Una ricercaintervento sull'educazione tra pari. Milano: FrancoAngeli.

Rogers, C. R. (1973). Libertà nell'apprendimento. Firenze: Giunti.

Šejtanićli, S., \& Ilić, M. (2016). Verbal Interaction betwen Pupils and Teachers in the Teaching Process. European Journal of Education, 2(8), 163-175.

Tian, L., Du, M., \& Huebner, E. S. (2015). The effect of gratitude on elementary school students' subjective well-being in schools: the mediating role of prosocial behavior. Social Indicators Research, 122(3), 887-904. DOI: 10.1007/s11205-014-0712-9.

Tschannen-Moran, M., \& Woolfolk Hoy, A. (2001). Teacher efficacy: capturing an elusive construct. Teaching and Teacher Education, 17, 783-805. DOI: 10.1016/S0742-051X(01)00036-1.V

Tschannen-Moran, M., Woolfolk Hoy, A., \& Hoy, W. K. (1998). Teacher efficacy: its meaning and measure. Review of Educational Research, 68(2), 202248. DOI: $10.3102 / 00346543068002202$.

Tsouloupas, C. N., Carson, R. L., \& Matthews, R. A. (2014). Personal and School Cultural Factors associated with the perceptions of Teachers' Efficacy in Handling Student Misbehavior. Psychology in the Schools, 51(2), 164-180. DOI: $10.1002 /$ pits. 21739.

Van Riel, C. B. M., \& Fombrun C. J. (2007), Essentials of Corporate Communication: Implementing Practices for Effective Reputation Management. Front Cover. UK: Taylor \& Francis.

Vecchione, M., Alessandri, G., Barbaranelli, C., \& Caprara, G. V. (2010). Higher-Order Factors of the Big Five and Basic. Values: Empirical and Theoretical Relations. British Journal of Psychology, 102, 478-498. DOI: 10.1111/j.20448295.2010.02006.x.

Watzlawick, P., Beavin, J. H., \& Jackson, D. D. (1971). Pragmatica della comunicazione umana. Roma: Astrolabio.

Woolfolk Hoy, A., \& Weinstein, C. S. (2006). Student and Teacher Perspectives on Classroom Management. In C. M. Evertson, \& C. S. Weinstein (Eds.), Handbook of Classroom Management. Research, Practice, and Contemporary Issues (pp. 181-219). Mahwah, NJ: Lawrence Erlbaum Associates.

Zhang, L. F. (2004). Thinking styles: university students' preferred teaching styles and their conceptions of effective teachers. The Journal of Psychology, 138(3), 233-252. DOI: 10.3200/JRLP.138.3.233-252.

Zimmerman, B. B., \& Paul, D. (2007). Technical communication teachers as mentors in the classroom: Extending an invitation to students. Technical Communication Quarterly, 16(2), 175-200. DOI: 10.1080/10572250709336559. 
Zeng, S., Benner, G. J., \& Silva, R. M. (2016). Effects of a summer learning program for students at risk for emotional and behavioral disorders. Education and Treatment of Children, 39(4), 593-615. DOI: 10.1353/etc.2016.0026. 